\title{
Neurocognitive Assessment
}

National Cancer Institute

\section{Source}

National Cancer Institute. Neurocognitive Assessment. NCI Thesaurus. Code C156775.

Tests to assess aspects of cognition, including attention, memory, language, reaction time, and perception. 